\title{
A novel ODT or chewable dosage form for acetaminophen: an isoleucine prodrug approach
}

\begin{abstract}
The physiochemical property of acetaminophen makes it difficult to be formulated as an orally disintegrating tablet (ODT) or chewable dosage form due to its unpleasantly bitter taste. Prodrug approach of acetaminophen may mask the bitterness of acetaminophen. Isoleucine ester prodrug of acetaminophen (Isoleucine-APAP) was synthesized and evaluated for its hydrolysis in PBS buffer at various $\mathrm{pH}$ values and in Caco-2 cell homogenate. Physicochemical characteristics such as melting point and stability of the Isoleucine-APAP prodrug were determined by MDSC technique. The results demonstrate that Isoleucine-APAP is more stable at lower $\mathrm{pH}$ than higher $\mathrm{pH}$ in PBS buffer. For example, the half-life $\left(\mathrm{T}_{1 / 2}\right)$ of Isoleucine-APAP is $200 \mathrm{~min}$ at $\mathrm{pH} 2.0$, but $158 \mathrm{~min}$ at $\mathrm{pH} 5.0$, and 4.34 min at $\mathrm{pH} 7.4$, respectively. When Carboxypeptidase-A was added to the PBS buffer at $\mathrm{pH}$ 7.4 , the $\mathrm{T}_{1 / 2}$ of Isoleucine-APAP was reduced to $2.30 \mathrm{~min}$. Furthermore, at $\mathrm{pH} 7.4$, the $\mathrm{T}_{1 / 2}$ of Isoleucine-APAP in a solution containing Caco-2 cell homogenate is only $0.682 \mathrm{~min}$, much shorter than that in the PBS buffer. This demonstrates that enzymes in the cell homogenate accelerate the hydrolysis of the ester bond. These data indicate that Isoleucine prodrug of acetaminophen may be a good candidate to be developed as ODT or chewable formulations.
\end{abstract}

Keywords: acetaminophen, isoleucine-APAP, isoleucine, prodrug, carboxypeptidase A, ODT, chewable dosage form
Volume 8 Issue 6 - 2020

\author{
Zhiqian Wu,' Pankit Bhavsar, ${ }^{2}$ Masumi Dave, ${ }^{3}$ \\ Xudong Yuan, ${ }^{4}$ Desuo Wang' \\ 'Department of Pharmaceutical Sciences, School of Pharmacy, \\ Philadelphia College of Osteopathic Medicine, USA \\ ${ }^{2}$ Amneal Pharmaceuticals, USA \\ ${ }^{3}$ Gattefossé Corporation, USA \\ ${ }^{4}$ iView Therapeutics, USA
}

\begin{abstract}
Correspondence: Zhiqian Wu, Department of Pharmaceutica Sciences, School of Pharmacy, Philadelphia College of Osteopathic Medicine, Suwanee, GA 30024, Tel 678-407-7366, Fax 678-407-4586, Email zhiqianwu@pcom.edu
\end{abstract}

Received: December 14, 2020 | Published: December 22, 2020

\section{Introduction}

Acetaminophen, also known as $N$-acetyl- $p$-aminophenol (APAP) or Paracetamol (outside of North America) is widely used for pain relief and antipyretic therapy in general population. Actually, acetaminophen is the most prescribed medication in children for the treatment of fever and pain. There are some pharmaceutical issues associated with acetaminophen such as its bitter taste and poor bioavailability. ${ }^{1,2}$ The unpleasantly bitter taste of acetaminophen poses significant challenges to formulate a suitable dosage form for pediatric use. Currently, flavoring with variety of fruity flavors to mask the bitter taste is the method used for this purpose. Modifying the bitterness with cation and anion salts was also tried. ${ }^{3,4}$ In the market, children's acetaminophen is formulated, in the form of oral suspension, orally disintegrating tablet (ODT), and chewable tablets. ${ }^{3}$ Even with the taste masking by adding various flavors there is a need for alternative approach to make it more appealing for pediatric use. Orally administered phenols typically show poor bioavailability and hepatic toxicity due to first-pass metabolism in the gastrointestinal tract and liver. ${ }^{5}$ These toxic effects have been attributed to the formation of a toxic metabolite $N$-acetylquinone imine, which is detoxified by reaction with glutathione leading to glutathione depletion and cell death. ${ }^{6}$ For many decades, pharmaceutical research has tried to solve these issues associated with acetaminophen by prodrug approaches. ${ }^{7-9}$ It was reported that the bitter taste could be masked by capping the hydroxyl group with promoieties including tetrahydropyran and ethoxyethan. ${ }^{7}$ Esterification of acetaminophen with amino acids and peptides (promoieties) was found to effectively overcome liver toxicity of the drug at high doses and significantly enhance aqueous solubility. ${ }^{8,9}$ Transdermal delivery is another valid approach to avoid first-pass metabolism. Drugs with phenolic functional groups are attractive candidates for topical drug delivery via prodrug approach. ${ }^{10-12}$ The phenolic $\mathrm{OH}$ group is very reactive and easy to attach a variety of promoieties. Most of the previous work on the phenols via a prodrug approach has focused on the corresponding ester or ether. ${ }^{13,14}$ Sulphate and amino acid esters of acetaminophen had been investigated and developed as potential prodrugs. ${ }^{15}$

In this study, we chemically attached amino acid isoleucine to acetaminophen and produced an isoleucine ester acetaminophen compound (Isoleucine-APAP). Isoleucine ester prodrugs were found to have good stability in aqueous solution. ${ }^{16}$ The physicochemical properties of Isoleucine-APAP were characterized. The purpose was to develop a prodrug of acetaminophen that could mask the bitter taste for formulation of ODT and provide a chewable dosage form of acetaminophen to pediatric patients. In addition, this prodrug might also be developed as a transdermal delivery medication to bypass the first- pass metabolism of acetaminophen and reduce its liver toxicity.

\section{Materials}

Acetaminophen, Boc-L-Isoleucine-OH, $N$-dimethylaminopyridine (DMAP), $N, N$ - dicyclohexylcarbodiimide (DCC), trifluoroacetic acid (TFA), Carboxypeptidase-A, other reagents and solvents were purchased from Sigma-Aldrich Chemical Company (Milwaukee, WI). HPLC grade methanol was purchased from Fisher Scientific Company (St. Louis, MO). Cell culture reagents were purchased from Invitrogen (Carlsbad, CA). Cell culture supplies were obtained from Corning (Corning, NY) and Falcon (Lincoln Park, NJ). All chemicals were either analytical or HPLC grade.

\section{Methods}

\section{Synthesis of isoleucine acetaminophen ester prodrug}

Isoleucine-APAP was synthesized by using a method described previously. ${ }^{17,18} 1.5$ eq. N- $t$ Boc-protected isoleucine was added dropwise into the mixture of 1.5 eq. DCC and 0.15 eq. DMAP and 1 eq. acetaminophen in dry DMF. The reaction was stirred at room temperature for $24 \mathrm{hr}$. and monitored by TLC (hexane:ethyl acetate, 1:1). After removing DMF under high vacuum, the residual mixture was extracted with ethyl acetate $(50 \mathrm{ml})$ and washed first with water $(2 \times 30 \mathrm{ml})$, then with saturated $\mathrm{NaHCO}_{3}(2 \times 30 \mathrm{ml})$, and saturated 
$\mathrm{NaCl}(1 \times 30 \mathrm{ml})$. The organic layer was dried over $\mathrm{MgSO}_{4}$, filtered, and concentrated. Crude compounds were separated using column chromatography (starting with hexane: ethyl acetate, 10:1). The fractions were collected and analyzed by TLC for purity. Fractions from each spot were concentrated under vacuum separately. The Boc protecting group was removed in $10 \mathrm{ml}$ of TFA: DCM (1:1) with stirring for $4 \mathrm{hr}$. After evaporating DCM and most TFA, cold ether was added to precipitate out pure product. After removing ether under vacuum, the product was reconstituted with water and lyophilized as white powder.<smiles>CCC(C)C(NC(=O)OC(C)(C)C)C(=O)Oc1ccc(NC(=O)OC(C)=O)cc1</smiles>

Scheme I Synthesis of Isoleucine-APAP.

The purity of Isoleucine-APAP was determined by HPLC analysis with the final product purity greater than $96 \%$. Isoleucine-APAP was easily separated from parent drug by HPLC method. Structural identity was confirmed using proton nuclear magnetic resonance spectra (1HNMR). 1HNMR spectra were obtained with a $400 \mathrm{MHz}$ Bruker NMR spectrometer. Electrospray ionization mass spectra (ESI-MS) were obtained on a Thermoquest LCQ electrospray ionization mass spectrometer. The observed molecular weight of the prodrug was found to be identical to that acquired from the target structure.

The synthesis method produced a 56\% yield of Isoleucine-APAP. The structure identity registered the following 1HNMR spectra (DMSO): 0.98 (t, 3H, isoleucine $\left.\mathrm{CH}_{3}\right), 1.09$ (d, 3H, isoleucine $\mathrm{CH}_{3}$ ), $1.50\left(\mathrm{~m}, 2 \mathrm{H}\right.$, isoleucine $\left.\mathrm{CH}_{2}\right), 2.11(\mathrm{~m}, 1 \mathrm{H}$, isoleucine $\mathrm{CH}), 2.10(\mathrm{~s}$, $\left.3 \mathrm{H}, \mathrm{CH}_{3}\right), 4.14(\mathrm{~d}, 1 \mathrm{H}, \alpha-\mathrm{H}), 7.11\left(\mathrm{~d}, 2 \mathrm{H}, \mathrm{CH}_{\text {benzine }}-\mathrm{O}\right), 7.62(\mathrm{~d}, 2 \mathrm{H}$, $\left.\mathrm{CH}_{\text {benzine }}-\mathrm{N}\right)$; ESI-MS: $264(\mathrm{M}+\mathrm{H})^{+}$.

\section{HPLC analysis}

Acetaminophen and Isoleucine-APAP ester were analyzed by reversed phase high performance liquid chromatography (RP-HPLC) performed at room temperature. Detection wavelength was $243 \mathrm{~nm}$. Samples were eluted at a flow rate of $0.6 \mathrm{ml} / \mathrm{min}$. The mobile phase consisted of $10 \% \mathrm{v} / \mathrm{v}$ Methanol and $1 \% \mathrm{v} / \mathrm{v}$ Glacial Acetic acid in deionized water. HPLC system was obtained from Water's corporation which consists of PDA detector-2998, separation module- 2695 alliance; column used was Water's XBridge ${ }^{\mathrm{TM}}$ shield RP18 $3.5 \mu \mathrm{m}$, $4.6 \times 150 \mathrm{~mm}$ and quick startEmpower 2 software. (Waters, Parsippany, NJ, USA).

\section{Hydrolysis assay of Isoleucine-APAP in PBS}

The chemical stability of the prodrug was determined in PBS at $\mathrm{pH}$ 2.0, 5.0, 7.4 and at $37^{\circ} \mathrm{C}$ to evaluate the non-enzymatic hydrolysis. ${ }^{18}$ The hydrolysis reactions were carried out in 96-well plates. PBS buffer $(230 \mu \mathrm{L})$ was placed in triplicate wells and the reactions were started with the addition of Isoleucine-APAP compound and incubated at $37^{\circ} \mathrm{C}$. At various time points, $40 \mu \mathrm{L}$ aliquots were removed and added to $40 \mu \mathrm{L}$ of $10 \%$ ice-cold TFA. The mixtures were centrifuged for $10 \mathrm{~min}$ at $2000 \mathrm{rpm}$ and $4^{\circ} \mathrm{C}$ and filtered through a $0.45 \mu \mathrm{m}$ filter. The filtrate was then analyzed by HPLC protocol described above.

\section{Hydrolysis of Isoleucine-APAP in Caco-2 cell homogenate and Carboxypeptidase-A}

The hydrolysis of Isoleucine-APAP was determined in Caco-2 cell line by methods described previously. ${ }^{18}$ The cell line was cultured at $37^{\circ} \mathrm{C}$ with $5 \% \mathrm{CO}_{2}$ and $90 \%$ relative humidity. Caco-2 cells were cultured in $80 \%$ minimum Essential Medium (MEM) with 20\% FBS. When the cells reached confluent condition, the culture was divided to $1: 4$ or 1:6 every 3-5 days using trypsin/EDTA solution. Cell homogenate were prepared when the cell cultures were $90 \%$ confluent. After trypsinization, the cells were washed three times with $\mathrm{pH} 7.4$ PBS buffer and re- suspended in $\mathrm{pH}$ 7.4 PBS $(10 \mathrm{mM})$. To prepare cell homogenate, $1 \%$ Triton-X 100 was added in PBS solution and vortexed vigorously. The cell suspension was centrifuged at 18,000 $\mathrm{rpm}$ for $30 \mathrm{~min}$ at $4^{\circ} \mathrm{C}$. The supernatant was used in Isoleucine-APAP hydrolysis assay. The total protein was quantified with the BioRad Protein Assay using bovine serum albumin as standard. The protein content was adjusted to approximately $1,000 \mu \mathrm{g} / \mathrm{ml}$ by appropriate dilutions before being used in hydrolysis studies. The hydrolysis of Isoleucine-APAP incubated with Carboxypeptidase- A was carried out by following the instruction of the enzyme kit.

\section{Melting point determination by MDSC technique}

Modulated Differential Scanning Calorimetry (MDSC) has many applications in pharmaceutical field from determination of melting point of compounds, confirmation of crystalline or amorphous nature of powder, to finding purity and polymorphism of samples. TA MDSC Q100 series was used to measure melting point of acetaminophen and Isoleucine-APAP by a method described previously. ${ }^{18}$ About $5 \mathrm{mg}$ of drug powder was sealed in aluminum hermetic pan with lid and loaded on MDSC to obtain the thermogram for the samples by setting the parameters as: equilibrate at $20.00^{\circ} \mathrm{C}$, Ramp $10.00^{\circ} \mathrm{C} / \mathrm{min}$ to $250.00^{\circ} \mathrm{C}$. The maximum temperature was modified as per the known theoretical melting point of sample to be analyzed.

\section{Results}

\section{Chemical hydrolysis of Isoleucine-APAP}

Chemical hydrolysis of Isoleucine-APAP was conducted in PBS buffer at three different $\mathrm{pH}$ values: $\mathrm{pH}$ 2.0, 5.0 and 7.4. Estimated halflives $\left(\mathrm{T}_{1 / 2}\right)$ of Isoleucine-APAP in phosphate buffers were calculated using linear regression analysis (Isoleucine-APAP concentrations vs. time) and the data points are listed in Table 1. In PBS pH 2.0 medium, $\mathrm{T}_{1 / 2}$ of Isoleucine-APAP was found to be $200.6 \mathrm{~min}$ which is the longest half-life in all media. In PBS pH 5.0 medium, $\mathrm{T}_{1 / 2}$ of Isoleucine-APAP decreased to $158.4 \mathrm{~min}$. While in PBS $\mathrm{pH} 7.4, \mathrm{~T}_{1 / 2}$ of the compound further reduced to $4.3 \mathrm{~min}$ (Figures 1-6).

Table I Half-life of Isoleucine-APAP in different hydrolysis media (mean \pm S.D.)

\begin{tabular}{llllll}
\hline Hydrolysis medium & Caco-2 cell homogenate & PBS pH 7.4 Carboxypeptidase A & PBS pH 7.4 & PBS pH 5.0 & PBS pH 2.0 \\
\hline Half-life (minutes) & $0.682 \pm 0.08$ & $2.30 \pm 0.15$ & $4.34 \pm 0.7$ & $158.37 \pm 2.1$ & $200.61 \pm 2.5$ \\
\hline
\end{tabular}




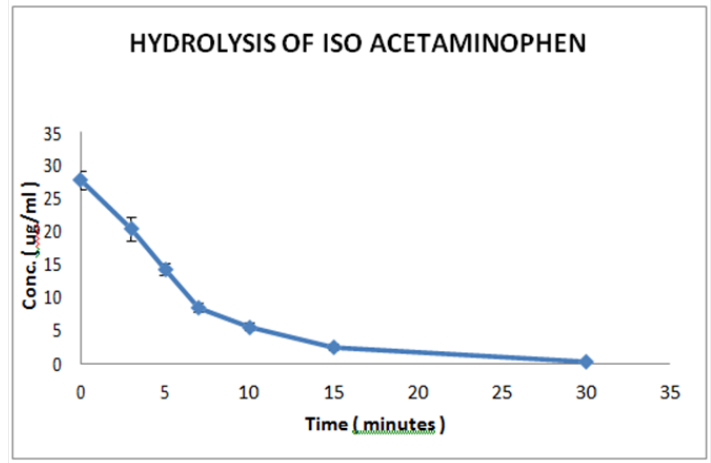

Figure I Hydrolysis of isoleucine-APAP in PBS pH 7.4 medium.

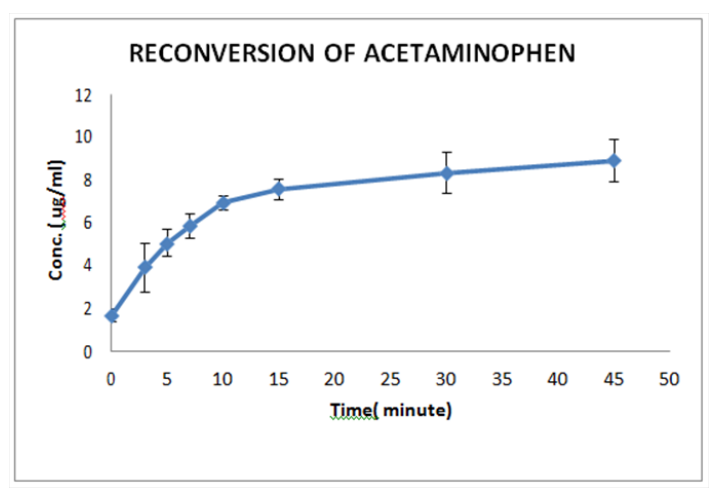

Figure 2 Reconversion of acetaminophen in PBS pH 7.4 medium.

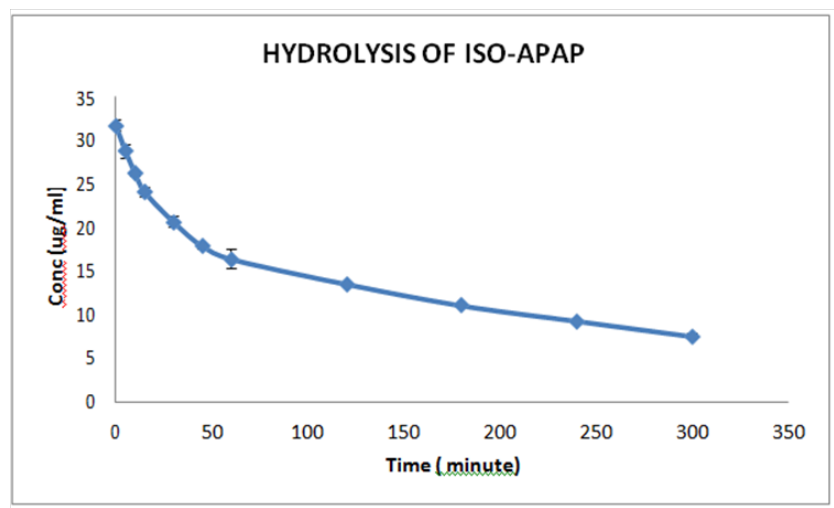

Figure 3 Hydrolysis of isoleucine-APAP in PBS pH 5.0 medium.

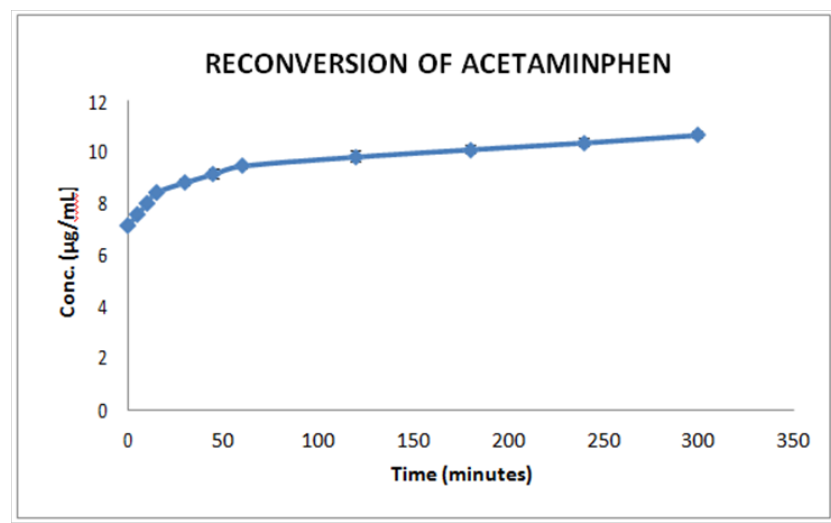

Figure 4 Reconversion of acetaminophen in PBS pH 5.0 medium.

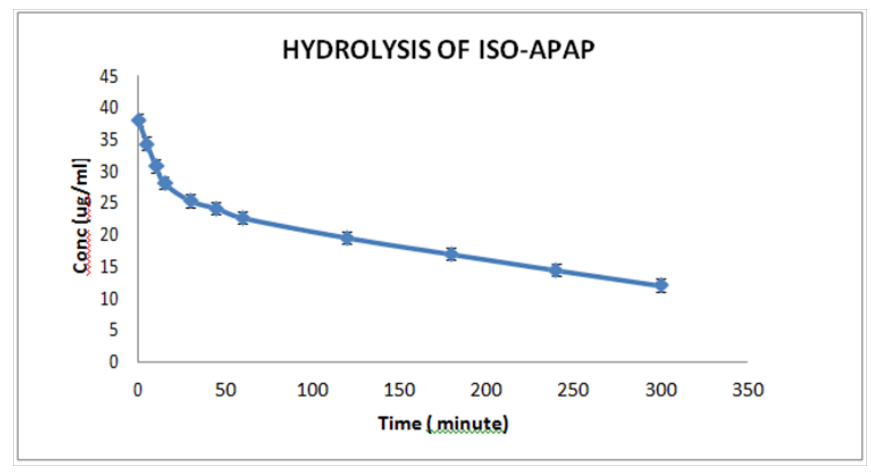

Figure 5 Hydrolysis of isoleucine-APAP in PBS pH 2.0 medium.

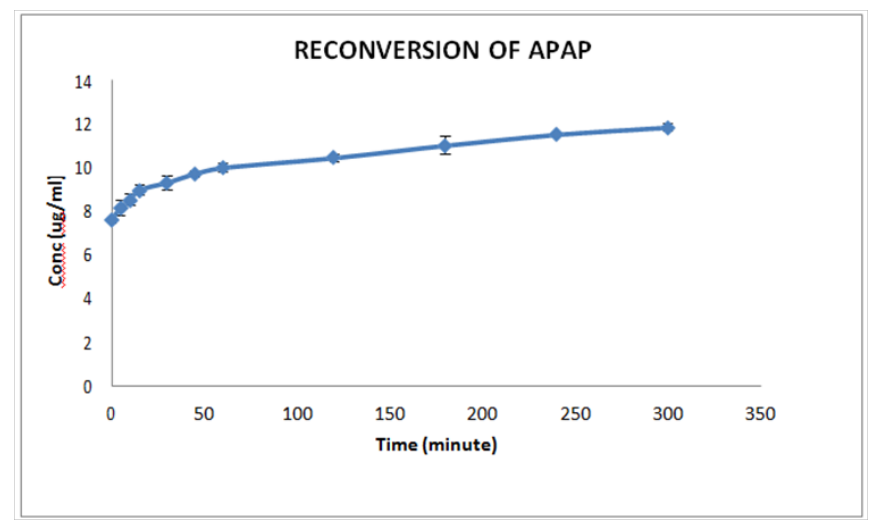

Figure 6 Reconversion of APAP in PBS pH 2.0 medium.

\section{Enzymatic hydrolysis of Isoleucine-APAP}

The half-lives of Isoleucine-APAP in Caco-2 cell homogenates and Carboxypeptidase-A was estimated using linear regression analysis (Isoleucine-APAP concentrations vs. time) and data are listed in Table 1. In Caco-2 cell homogenate, $T_{1 / 2}$ of Isoleucine-APAP was found to be only $0.7 \mathrm{~min}$. While incubated with carboxypeptidase-A, $\mathrm{T}_{1 / 2}$ of Isoleucine-APAP was about $2.3 \mathrm{~min}$. In comparison, Isoleucine-APAP hydrolysis rate was significantly faster in Caco-2 cell homogenates than in phosphate buffer at $\mathrm{pH}$ 7.4. This indicates that the enzymes in the epithelial cells of small intestine play an important role in the breakdown of Isoleucine-APAP and reconversion of acetaminophen (Figures 7-10).

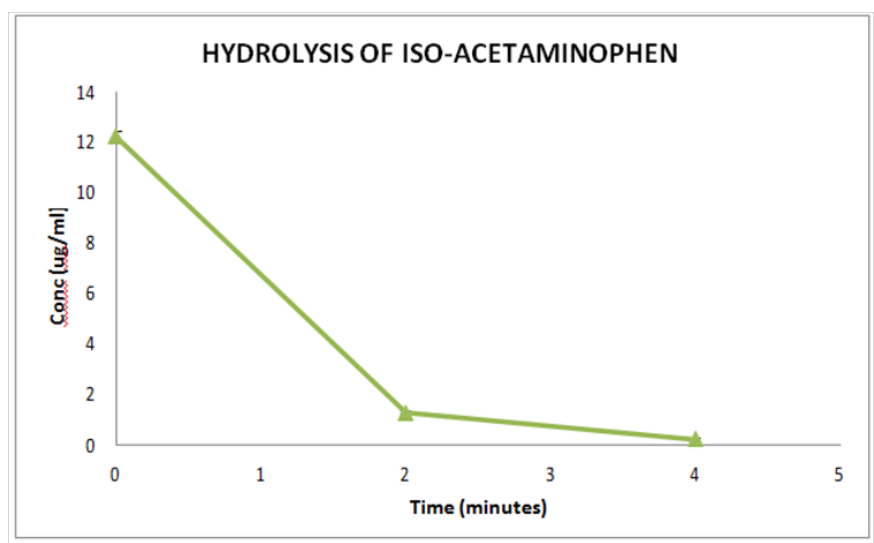

Figure 7 Hydrolysis of Isoleucine-APAP in Caco-2 cell homogenate. 


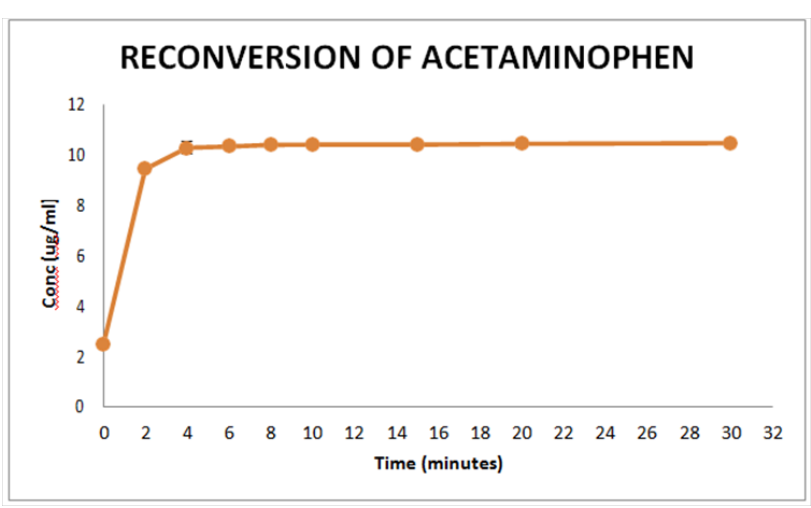

Figure 8 Reconversion of Acetaminophen in Caco-2 cell homogenate.

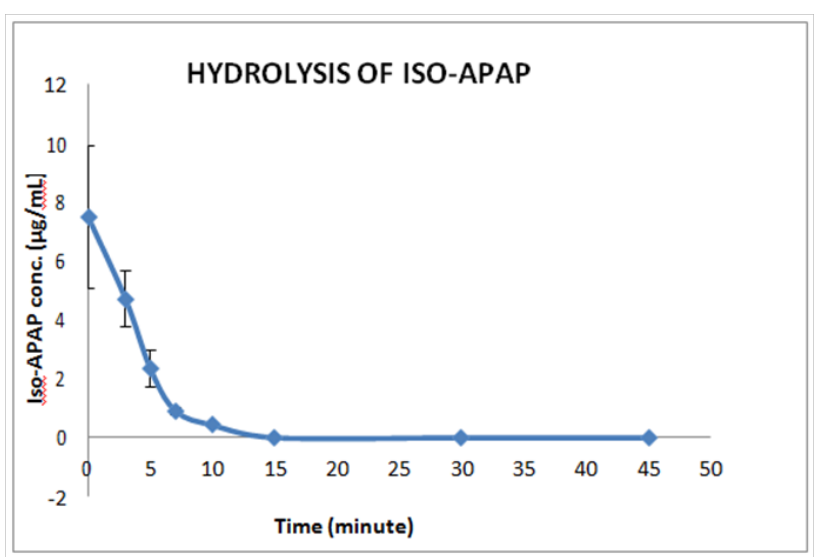

Figure 9 Hydrolysis of Isoleucine-APAP in PBS pH 7.4 + Carboxypeptidase A.

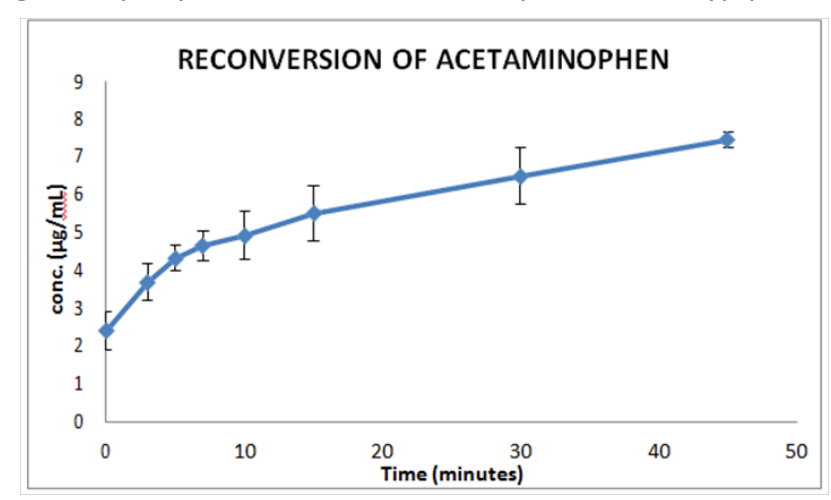

Figure I0 Reconversion of Acetaminophen in PBS pH 7.4 + Carboxypeptidase A.

\section{Melting point determination}

Modulated DSC (MDSC) is modification of conventional DSC which provides additional information for the compound of interest. Differential scanning calorimetry (DSC) is a thermal analysis technique that has been used to measure thermodynamic properties associated with transitions in materials as a function of time and temperature. MDSC was used for physical characterization of Acetaminophen and Isoleucine-APAP. MDSC thermograms of these samples were recorded from 20 to $240^{\circ} \mathrm{C}$. Melting points of Acetaminophen and Isoleucine-APAP were determined. Acetaminophen shows characteristic endothermic peak at $171^{\circ} \mathrm{C}$ as it has been reported. In the meantime, isoleucine prodrug shows endothermic peak at $122^{\circ} \mathrm{C}$ (Figures 11-13).

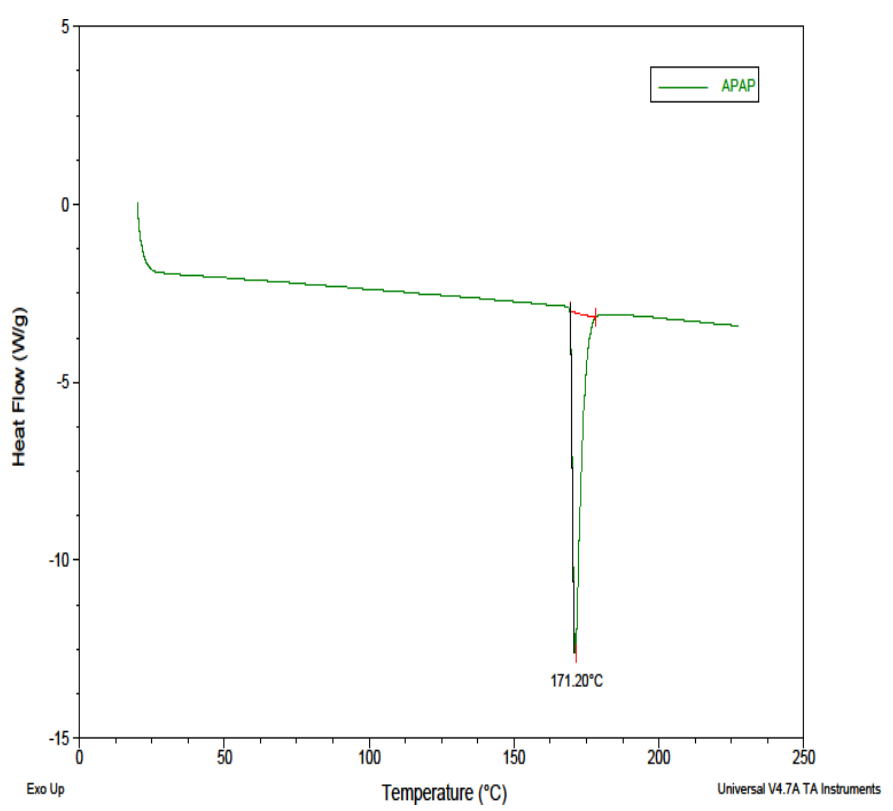

Figure I I DSC thermogram of Acetaminophen.

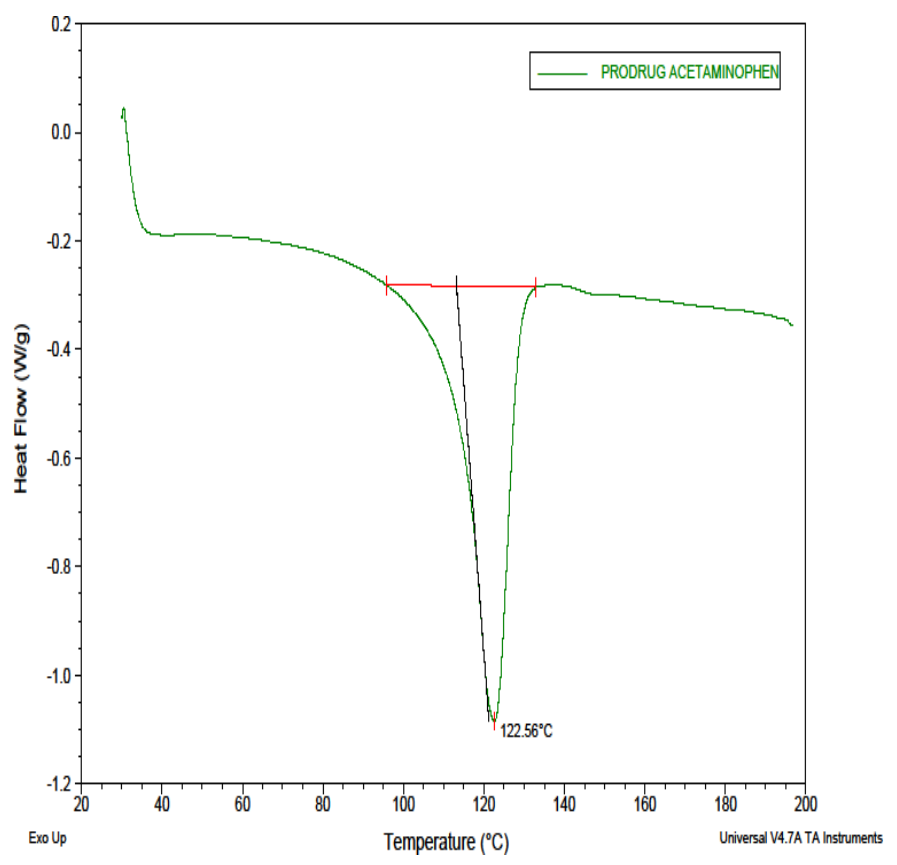

Figure 12 DSC thermogram of Isoleucine-APAP.

\section{Discussion}

In this study, a potential prodrug (Isoleucine-APAP) was synthesized by esterification of the hydroxyl group in acetaminophen. The esterification is a commonly used strategy to design prodrugs. ${ }^{16-18}$ The primary purpose of this study is to develop an acetaminophen prodrug which is suitable to be formulated as chewable or ODT dosage forms by masking the bitter taste of acetaminophen. We found that the chemical stability of Isoleucine-APAP was suitable to serve this purpose. The ester structure of Isoleucine-APAP is a relatively stable in lower $\mathrm{pH}$ solution and even when the $\mathrm{pH}$ value was increased to 7.4 , the compound still has a $\mathrm{T}_{1 / 2}$ greater than four minutes. This duration is more than enough for ODT or chewable dosage forms. The 
breakdown of the compound in the presence of digestive enzymes was greatly accelerated in assay condition containing the homogenate of Caco-2 cell, which indicates that the Isoleucine-APAP ester compound can be easily reconverted back to its parent drug acetaminophen in the digestive and absorption site (i.e., small intestine). These properties make the newly synthesized Isoleucine- APAP compound an appealing candidate to be developed as a prodrug for acetaminophen especially for pediatric patient population.

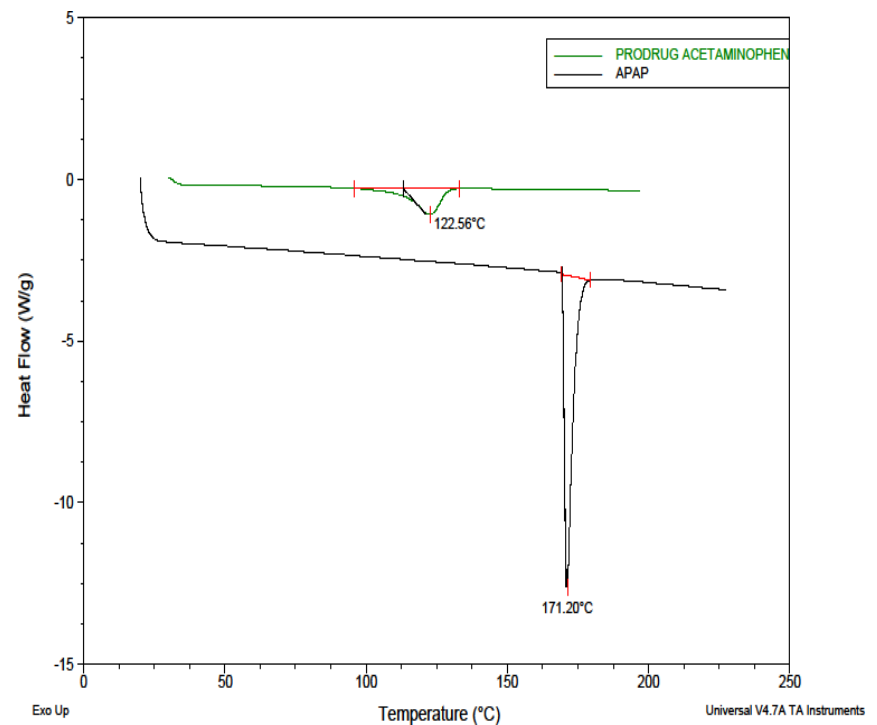

Figure I 3 A DSC thermogram overlay of Acetaminophen and IsoleucineAPAP.

Although saliva contains some esterase that may cause hydrolysis of Isoleucine-APAP, the esterase activity is relatively low and the saliva flow rate is four folds slow at rest. ${ }^{19}$ The assay data indicate that Isoleucine-APAP ester is more stable at lower $\mathrm{pH}$ than higher $\mathrm{pH}$, which is consistent with the stability of other amino acid ester prodrugs such as Valacyclovir and Ile-gemcitabine. ${ }^{17}$ The normal saliva $\mathrm{pH}$ is in a range of 6.5 to 7.4 . This suggests that Isoleucine-APAP might be suitable to be formulated into a chewable tablet to improve acetaminophen's administration to young kids. The current study is a conceptual and feasibility exploration. Whether the Isoleucine-APAP compound can mask the bitterness of acetaminophen, a human study needs to be carried out eventually. The fact that the phenol group of acetaminophen, which may contribute to the bitter taste, is capped by isoleucine-a natural amino acid, makes this prodrug approach less bitterness than acetaminophen and is suitable for ODT and chewable dosage forms.?

Carboxypeptidase A was incubated with Isoleucine-APAP in PBS buffer at $\mathrm{pH} 7.4$ with half-life of $2.3 \mathrm{~min}$, which is very close to $50 \%$ of the half-life in buffer itself. This indicates that peptidases contribute to the hydrolysis of Isoleucine-APAP. The half-life of Isoleucine-APAP in Caco-2 cell homogenate is less than $1 \mathrm{~min}$, much shorter than the half-life in PBS buffer at pH 7.4 and the half-life in the solution containing carboxypeptidase $\mathrm{A}$. These results indicate other enzymes in the cell homogenate also contribute to the hydrolysis of the ester bond. These enzymes could be esterases and peptidases, which are known to hydrolyse ester bond. It was reported that Caco-2 cells express both esterases and peptidases..$^{20,21}$

Isoleucine ester prodrugs have longer half-lives compared with other amino acids ester prodrugs, such as proline ester prodrugs. ${ }^{17}$ In our previous study, we reported that proline prodrug design of
APAP has a half-life of $120 \mathrm{~min}$ at $\mathrm{pH} 2.0$, much shorter than the half-life of Isoleucine-APAP about 200 minutes) at the same $\mathrm{pH} .{ }^{18}$ The half-life of Isoleucine-APAP is about $4.3 \mathrm{~min}$ in PBS buffer at $\mathrm{pH} 7.4$, which is appropriate for ODT and chewable dosage forms. After chewing and dissolving, the prodrug is expected to be quickly converted to acetaminophen and absorbed rapidly in small intestines. Moreover, any leftover prodrug can easily covert to acetaminophen in the small intestine with higher $\mathrm{pH}$ and various enzymes. IsoleucineAPAP synthesized here is in the form a TFA salt. To be formulated as a chewable dosage form or ODT to mask the bitter taste of acetaminophen, the TFA salt should be converted to HCL salt first.

\section{Acknowledgments}

None.

\section{Conflicts of interest}

Authors declare that there is no conflict of interest.

\section{References}

1. Suzuki H, Onishi H, Takahashi Y, et al. Development of oral acetaminophen chewable tablets with inhibited bitter taste. Int J Pharm. $2003 ; 251(1-2): 123-132$.

2. Hejaz H, Karaman R, Khamis M. Computer-assisted design for paracetamol masking bitter taste prodrugs. JMol Model. 2012;18(1):103114

3. Autret E, Breart G, Jonville AP, et al. Comparative efficacy and tolerance of ibuprofen syrup and acetaminophen syrup in children with pyrexia associated with infectious diseases and treated with antibiotics. Eur $J$ Clin Pharmacol. 1994;46(3):197-201.

4. Keast RS, Breslin PA. Modifying the bitterness of selected oral pharmaceuticals with cation and anion series of salts. Pharm Res. 2002;19(7):1019-1026.

5. Boyer TD, Rouff SL. Acetaminophen-induced hepatic necrosis and renal failure. JAMA. 1971;218(3):440-441.

6. Vermeulen NP, Bessems JG, Van de Straat R. Molecular aspects of paracetamol-induced hepatotoxicity and its mechanism-based prevention. Drug Metab Rev. 1992;24(3):367-407.

7. Repta AJ, Hack J. Acetaminophen prodrugs: 2-(p-acetaminophenoxy) tetrahydropyran. J Pharm Sci. 1973;62(11):1892-1894.

8. Hussain A, Kulkarni P, Perrier D. Prodrug approaches to enhancement of physicochemical properties of drugs IX: acetaminophen prodrug. $J$ Pharm Sci. 1978;67(4):545-546.

9. Santos C, Mateus MI, Santos AP, et al. Cyclization-activated prodrugs. Synthesis, reactivity and toxicity of dipeptide esters of paracetamol. Bioorganic \& Medicinal Chemistry Letters. 2005;15(6):1595-1598.

10. Thomasa JD, Sloan KB. In vitro evaluation of alkylcarbonyloxymethyl (ACOM) ethers as novel prodrugs of phenols for topical delivery: ACOM prodrugs of acetaminophen. Int J Pharm. 2008;346(1-2):80-88.

11. Wasdo SC, Sloan KB. Topical delivery of a model phenolic drug: alkyloxycarbonyl prodrugs of acetaminophen. Pharm Res. 2004;21(6):940-946.

12. Majumdar S, Sloan KB. Synthesis, hydrolyses and dermal delivery of $\mathrm{N}$-alkyl-N- alkyloxycarbonylaminomethyl (NANAOCAM) derivatives of phenol, imide and thiol containing drugs. Bioorg Med Chem Lett. 2006;16(13):3590-3594.

13. Majumdar S, Sloan KB. Synthesis and topical delivery of N-alkyl$\mathrm{N}$-alkyloxycarbonylaminomethyl prodrugs of a model phenolic drug: Acetaminophen. International Journal of Pharmaceutics. 2007;337(12):48-55. 
14. Kovach IM, Pitman IH, Higuchi T. Amino acid esters of phenols as prodrugs: synthesis and stability of glycine, beta-aspartic acid, and alpha-aspartic acid esters of p-acetamidophenol. J Pharm Sci. 1981;70(8):881-885.

15. Williams DB, Varia SA, Stella VJ, et al. Evaluation of prodrug potential of sulphate ester of acetaminophen and 3-hydroxymethyl-phenytoin. International Journal of Pharmaceutics. 1983;14(1):113-120.

16. Song X, Lorenzi PL, Landowski CP, et al. Amino acid ester prodrugs of the anticancer agent gemcitabine: synthesis, bioconversion, metabolic bioevasion, and hPEPT1-mediated transport. Mol Pharm. 2005;2(2):157-167.

17. Vig BS, Lorenzi PJ, Mittal S, et al. Amino acid ester prodrugs of floxuridine: synthesis and effects of structure, stereochemistry, and site of esterification on the rate of hydrolysis. Pharm Res. 2003;20(9):13811388.
18. Wu Z, Patel A, Dave R, et al. Development of acetaminophen proline prodrug. Bioorg Med Chem Lett. 2010;20(13):3851-3854.

19. Hansen LB, Christrup LL, Bundgaard H. Saliva-catalyzed hydrolysis of a ketobemidone ester prodrug: Factors influencing human salivary esterase activity. International Journal of Pharmaceutics. 1992;88(13):221-227.

20. Basson MD, Hong F, Emenaker NJ. Specific modulation of intestinal epithelial brush border enzyme expression by a phorbol ester. J Surg Res. 59(1):121-126.

21. Imai $\mathrm{T}$, Imoto $\mathrm{M}$, Sakamoto $\mathrm{H}$, et al. Identification of esterases expressed in Caco-2 cells and effects of their hydrolyzing activity in predicting human intestinal absorption. Drug Metab Dispos. 2005;33(8):11851190. 